\title{
Two Cases of Gastroduodenal Perforation Following Cesarean Section
}

\author{
Ronald Awoonor-Williams ${ }^{\mathrm{a}}$, Cornelius Turpin ${ }^{\mathrm{a}, \mathrm{b}}$, Joseph Yorke, ${ }^{\mathrm{a}, \mathrm{c}}$, Wilfred Sam-Awortwi Jnr ${ }^{\mathrm{d}}$, \\ Michael Ofoe Adinkua, Anita Eseenam Agbeko ${ }^{a}$, Kwabena Agbedinu, \\ Steven Ansah Asamoahe, Sylvia Vanderpuye ${ }^{\mathrm{e}}$, Philip Agyemang Prempeh ${ }^{\mathrm{e}}$, \\ Regina Asantef ${ }^{f}$ Dorcas Ahulud, Dennis Afful Yorkeg, \\ Emmanuel Acheampongh, i
}

\begin{abstract}
Peptic ulcer disease with its complications in recent centuries has become a disease of global burden associated with high morbidity and mortality. There are several risk factors that have been implicated. These include Helicobacter pylori infection, regular use of non-steroidal anti-inflammatory drugs (NSAIDS), smoking, alcohol use, steroid use, male gender and low socioeconomic status. The ulcer can be either acute or chronic. Acute ulcers are mostly stress ulcerations with documented risk factors. Bleeding, and rarely perforation are the main complications which remain a surgical challenge. Some underlying causes of gastroduodenal perforation have been reported but remain largely rare. These include gastric carcinoma, ingestion of sharp foreign material, opportunistic infections, metastatic cancer, eating disorders and pregnancy. It is in line with these rare causes that we report two cases of gastroduodenal perforation post cesarean section in two young women, both succumbing to complications of
\end{abstract}

Manuscript submitted March 8, 2019, accepted March 25, 2019

${ }^{a}$ General Surgery Unit, Directorate of Surgery, Komfo Anokye Teaching Hospital, Kumasi, Ghana

bepartment of Obstetrics and Gynaecology, School of Medical Sciences, College of Health Sciences, Kwame Nkrumah University of Science and Technology, Kumasi, Ghana

'Department of Surgery, School of Medical Sciences, College of Health Sciences, Kwame Nkrumah University of Science and Technology, Kumasi, Ghana

${ }^{\mathrm{d}}$ Directorate of Anaesthesia and Intensive Care, Komfo Anokye Teaching Hospital, Kumasi, Ghana

eDirectorate of Obstetrics and Gynaecology, Komfo Anokye Teaching Hospital, Kumasi, Ghana

fDirectorate of Ear Nose and Throat, Komfo Anokye Teaching Hospital, Kumasi, Ghana

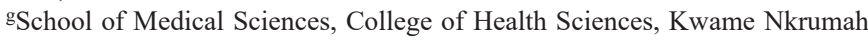
University of Science and Technology, Kumasi, Ghana

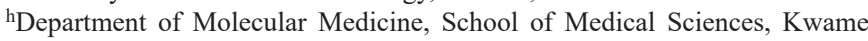
Nkrumah University of Science and Technology, Kumasi, Ghana

${ }^{\mathrm{i} C}$ Corresponding Author: Emmanuel Acheampong, Department of Molecular Medicine, School of Medical Sciences, Kwame Nkrumah University of Science and Technology, Kumasi, Ghana.

Email: emmanuelachea1990@yahoo.com

doi: https://doi.org/10.14740/jmc3279 the perforation.

Keywords: Gastroduodenal perforation; Peptic ulcer; Cesarean section

\section{Introduction}

Peptic ulcer disease (PUD) with its complications in recent centuries has become a disease of global burden associated with high morbidity and mortality, and significant geographical variation with respect to its prevalence [1,2]. Recent advances in its management with the advent of endoscopy and proton pump inhibitors, as well as the eradication Helicobacter pylori have led to a decrease in its incidence. However, its complications remain a challenge $[1,3,4]$.

Gastroduodenal perforation is potentially fatal with a significant mortality rate [5-7]. It affects about $2-10 \%$ of peptic ulcer disease patients [8]. This complication has a higher incidence in developing countries, affecting the younger generation $[9,10]$. The perforation leads to leakage of gastric or duodenal content into the peritoneal cavity which results in chemical peritonitis manifesting as generalized peritonitis. The peritonitis results in cardiovascular, renal, metabolic, respiratory and systemic effects which can potentially be fatal [11]. The duration of the perforation prior to surgical intervention is an independent determinant of outcome $[10,12]$. Treatment involves aggressive resuscitation of patients using crystalloids, blood products to correct the hematological, biochemical and physiological derangements which is followed by surgical intervention [13].

Some other underlying causes of gastroduodenal perforation have been reported but remain largely rare. These include gastric carcinoma, ingestion of sharp foreign material, opportunistic infections, metastatic cancer, eating disorders and pregnancy [14-16]. We report two cases of gastroduodenal perforation diagnosed at laparotomy on the seventh (case 1) and 12th (case 2) day post cesarean section in two young women. Both patients died from complications of the pathology on the seventh (case 1) and 16th (case 2) day post laparotomy for the 
Table 1. Patient's Renal Functional Results During the Period of Admission

\begin{tabular}{|c|c|c|c|c|c|c|c|c|c|}
\hline RFT & DA 1 & DA 2 & POD 1 & POD 2 & POD 3 & POD 4 & POD 5 & POD 6 & POD 7 \\
\hline Urea $(\mathrm{mmol} / \mathrm{L})$ & 56.62 & 65.81 & & & 49.93 & 50.25 & 20.59 & 41.04 & \\
\hline Creatinine $(\mu \mathrm{mol} / \mathrm{L})$ & 1,061 & 1,405 & & & 952 & 791 & 669 & 548 & \\
\hline $\mathrm{K}^{+}(\mathrm{mmol} / \mathrm{L})$ & 6.2 & 7.0 & 6.9 & 8.6 & 7.5 & 4.6 & 3.2 & 3.0 & \\
\hline $\mathrm{Cl}^{-}(\mathrm{mmol} / \mathrm{L})$ & 100 & 105 & 114 & 117 & 118 & 118 & 122 & 121 & \\
\hline
\end{tabular}

RFTs: renal function tests; DA: day of admission; POD: postoperative day.

closure of the perforations.

\section{Case Reports}

\section{Case 1}

A 25-year-old para 4 (all alive with one termination of pregnancy) woman, was referred from a peripheral hospital on account of jaundice and lower abdominal pain post cesarean section. The indication for the cesarean section was one previous cesarean section with pregnancy-induced hypertension (PIH) at term. On postoperative day 2 she became jaundiced. On postoperative day 5 she developed sudden onset of severe lower abdominal pain and was referred to Komfo Anokye Teaching Hospital (KATH) for further management.

There was no history of cola-like urine, abdominal distension, fever, discharge from the surgical wound or bleeding per vaginam. She had absolute constipation since the cesarean section. Past medical and surgical history was negative for hepatitis B infection, sickle cell disease and glucose-6-phosphate dehydrogenase (G6PD) deficiency. Her third pregnancy was 2.5 years ago and delivered by cesarean section on account of antepartum hemorrhage at term. She received antimalarials, antibiotics, analgesic (non-steroidal anti-inflammatory drugs (NSAIDS) and opiates) and four units of blood products prior to her referral.

At presentation to KATH, she was conscious, afebrile, jaundiced and pale. She had a pulse of 88 beats per minute, regular and of good volume, and blood pressure of 120/80 mm Hg.

Chest examination was unremarkable. Abdominal examination revealed a clean and dry Pfannenstiel incision with tenderness, guarding and rebound tenderness in both lower quadrants. Uterine size was approximately 20 weeks. Urine output as recorded from the referral site was scanty. That recorded after admission was less than $30 \mathrm{~mL} / \mathrm{h}$.

Blood was taken for full blood count, renal function test, grouping and cross matching and blood culture. Abdominal ultrasound showed moderate free intraperitoneal fluid with low level internal debris and septations, an approximately 18 -week size uterus with normal endometrium. The spleen, liver and the kidneys were normal. Blood investigations revealed severe derangements (Table 1).
Resuscitation was with intravenous (IV) crystalloids, blood products, and antibiotics (IV ceftriaxone $2 \mathrm{~g}$ daily and IV metronidazole $500 \mathrm{mg} 8$-hourly). The patient was managed by a team of obstetricians, surgeons, renal physician and anesthesiologist. Twenty-four $h$ after admission she became febrile with a temperature of $38.1^{\circ} \mathrm{C}$, respiratory rate of 36 cycles per minute with flaring of the alae nasi and oxygen saturation less than $90 \%$ in room air. The diagnoses she was being treated for were; generalized peritonitis secondary to iatrogenic bowel perforation post cesarean section (to rule out typhoid ileal perforation), severe sepsis and acute kidney injury with hyperkalemia.

She maintained urine output at about $35 \mathrm{~mL} / \mathrm{h}$ prior to surgery; treatment of hyperkalemia was instituted with calcium gluconate, soluble insulin and salbutamol nebulization.

Exploratory laparotomy was undertaken on $48 \mathrm{~h}$ of admission by a consultant general surgeon. Intraoperative findings were as follows; approximately $5 \mathrm{~L}$ of bilious fluid in the peritoneal cavity, a $1 \times 1 \mathrm{~cm}$ prepyloric gastric perforation on the anterior aspect of the lesser curvature of the stomach and petechial hemorrhages in the mucosa of the stomach, extensive fibrinous adhesions between small bowel and normal looking but bulky uterus, approximately 18 weeks in size.

Closure of the perforation was done by a modified Graham's omental patch and the peritoneum lavaged copiously with $5 \mathrm{~L}$ of warm saline. The patient was nursed postoperatively in the intensive care unit. She developed several complications within the postoperative period.

The preexisting acute kidney injury with its associated electrolyte imbalance persisted (Table 1). She also developed bleeding diathesis and severe sepsis. She succumbed to these complications on postoperative day 7 .

\section{Case 2}

Twenty-six years old woman, gravida 3 para 0 alive with two terminations of pregnancy who was referred from a peripheral hospital on account of high blood pressure at 40 weeks plus 5 days of gestation in labor. She was a regular attendant of antenatal clinic. Her past medical history revealed symptoms of dyspepsia 1 year prior to the index pregnancy which persisted during the pregnancy and treated with antacids.

On examination she was conscious, afebrile, anicteric and 
not pale. There was no pedal edema. Pulse was 98 beats per minute, regular and of good volume and blood pressure was 168/98 mm Hg. Chest examination was unremarkable. Abdominal examination revealed a symphysis-fundal height of $38 \mathrm{~cm}$ compatible with gestational age, longitudinal lie with cephalic presentation and a descent of $4 / 5$. The fetal heart rate was 138 beats per minute. Contractions were three in $10 \mathrm{~min}$ each lasting $35 \mathrm{~s}$. There was no abdominal tenderness.

On vaginal examination, cervical dilation was $6 \mathrm{~cm}$ with station of minus one. The examining finger was stained with grade 1 meconium liquor. Urine protein was trace on dipstick urinalysis.

An initial diagnosis of nullip with mild pre-eclampsia at 40 weeks plus 5 days in active phase of labor was made.

She was admitted for continuous monitoring of the progress of labor. Treatment included (tablet methyldopa $250 \mathrm{mg}$ 8 -hourly) with serial monitoring of the blood pressure.

Twelve hours after presentation, she developed epigastric pains. The blood pressure was 170/90 $\mathrm{mm} \mathrm{Hg}$. On abdominal examination, symphysis-fundal height was $38 \mathrm{~cm}$ compatible, longitudinal lie with cephalic presentation and a descent of $4 / 5$. Fetal heart rate was $156 \mathrm{bpm}$ and contractions were three in $10 \mathrm{~min}$ each lasting $40 \mathrm{~s}$. There was mild epigastric tenderness. On vaginal examination cervical dilatation was $8 \mathrm{~cm}$, station of plus one, moulding one plus and moderate caput. Urine protein was trace on dipstick urinalysis. A diagnosis of a nullip with imminent eclampsia was made, and she was prepared for emergency cesarean section.

Intraoperatively a live female baby weighing of $3.5 \mathrm{~kg}$ was delivered with Apgar scores of $6 / 10$ and $7 / 10$ in the first and fifth minute respectively. There was a normal looking gravid uterus and adnexa. The patient was nursed at a high dependency unit. She was on antibiotics and analgesics (IV Coamoxiclav 1.2 g 8-hourly, IV metronidazole 500 mg 8-hourly, suppository diclofenac $100 \mathrm{mg}$ 12-hourly and intramuscular (IM) pethidine $50 \mathrm{mg}$ 6-hourly). Her blood pressure dropped to $115 / 70 \mathrm{~mm} \mathrm{Hg}$ on postoperative day 2 without the antihypertensive. She was discharged on postoperative day 3 with a blood pressure of 120/70 mm Hg on oral co-amoxiclav $625 \mathrm{mg}$ bid for 7 days and metronidazole $400 \mathrm{mg}$ tid for 7 days, and suppository diclofenac $100 \mathrm{mg}$ bid for 5 days to be reviewed on weekly basis on the outpatient department.

Nine days after discharge she was seen at the hospital, having defaulted first review with a complaint of lower abdominal pain. There was no history of fever, vomiting, or bleeding per vaginam. She had not passed stools since the surgery and the lochia was pink and non-offensive.

The abdomen on examination was full of a clean dry surgical scar. There was tenderness in hypogastric region and bowel sounds were present. Vaginal examination findings were unremarkable. Her repeat laboratory investigation (Table 2) showed marked derangement in renal function.

Ultrasound revealed free intraperitoneal fluid with low level internal echoes respectively and the guided paracentesis yielded pus. A diagnosis of intraabdominal abscess post cesarean section was made.

Resuscitation was instituted and with IV fluids. Urine output was about $40 \mathrm{~mL} / \mathrm{h}$. She was also started on broad spectrum antibiotics. Exploratory laparotomy was done within 24

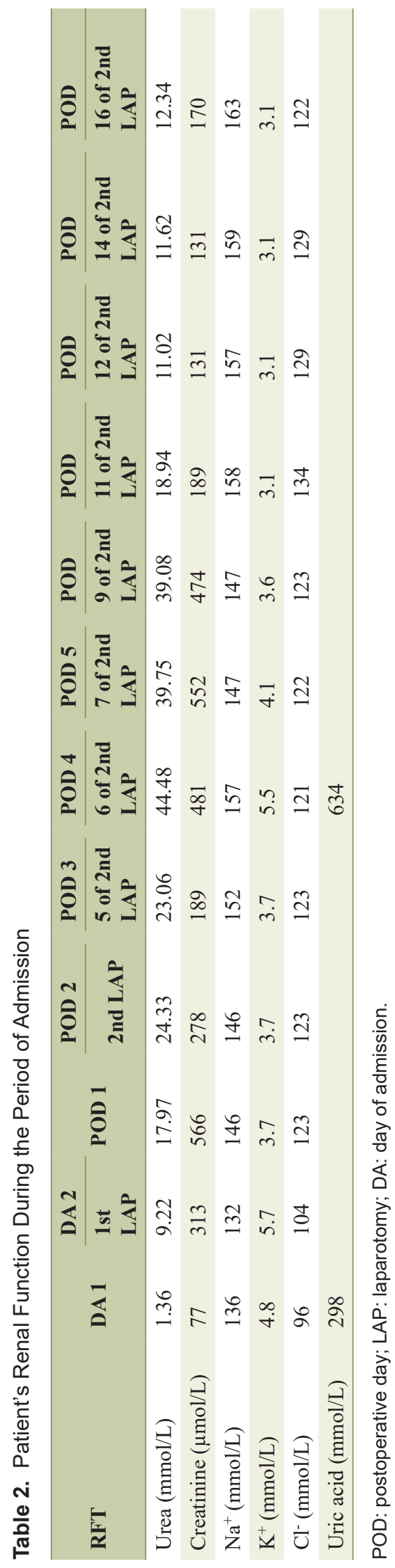


$\mathrm{h}$ of her second admission by a senior specialist obstetrician gynecologist.

Access was via the previous Pfannenstiel scar. Intraoperatively there was about $1 \mathrm{~L}$ of pus within the pelvic cavity, the uterus and adnexa were normal as well as the intestines and appendix. The pelvis was lavaged with $4 \mathrm{~L}$ of warm saline and the abdomen closed leaving a drain in the pelvis. She was nursed in the high dependency unit after the surgery where the antibiotics and IV fluids were continued. On postoperative day 2 she was noticed to be semiconscious with a Glasgow Coma Scale score of 11/15 (M5E3V3), urine output was less than 30 $\mathrm{mL} / \mathrm{h}$ and abdominal drain effluent was bilious.

A diagnosis of an enterocutaneous fistula was made and the patient prepared for another exploratory laparotomy after resuscitation to correct her physiological, hematological and biochemical abnormalities with IV fluids and blood products. Her urine output was $38 \mathrm{~mL} / \mathrm{h}$. A general surgery consult was sought, and the laparotomy was done on the same day.

Intraoperatively there was $1 \mathrm{~cm}$ (in diameter) perforation on the anterior aspect of the second part of the duodenum, $3 \mathrm{~L}$ of bilious fluid in the peritoneal cavity and fibrinous adhesion between edematous small bowel. Closure of the perforation was done followed by Graham's omental patch and the abdomen lavaged with $5 \mathrm{~L}$ of warm saline.

She was nursed at the intensive care unit. She developed several complications postoperatively which included severe sepsis, acute kidney injury and a high output enterocutaneous fistula. The closure broke down on postoperative day 5. The fistula was a high output type with its attendant electrolyte imbalances (from postoperative day 5 of Table 2) and malnutrition. Her renal impairment worsened (postoperative day 6 of Table 2) but was aggressively tackled via hemodialysis. However, shortage of blood products and parenteral nutritional products to correct these deficits significantly delayed another surgical intervention. She died on postoperative day 16 from sepsis. Sequential organ failure assessment (SOFA) scores computed in the ICU with these derangements and clinical parameters ranging from 6 to 9 from admission to demise.

\section{Discussion}

Complications of peptic ulcer disease are rare in pregnancy due to hormonal influences including progesterone and increased plasma histamine levels $[17,18]$. Its occurrence after cesarean section warrants further evaluation. There are several possible explanations to the pathogenesis from the etiological factors.

The use of NSAIDS in postoperative pain control may be contributory factor to the perforation. NSAIDS have been shown to have toxic effect on the mucosa [19] and their ingestion in the shortest duration can lead to acute ulceration and possible perforation [20,21]. Both patients were on analgesics with diclofenac an NSAID, as a component. Critically ill patients as exemplified in these two cases coupled with the stress of surgery may also be a contributory factor [20,22]. The presence of acute kidney injury (in both cases) and jaundice (in the first case) are known etiological factors to the development of acute ulceration and its progression to perforation [20]. The re- activation of preexisting ulcer during critical illness is another possibility which usually leads to bleeding. We are limited by the lack of possible endoscopic diagnosis of a preexisting ulcer in the second case. However, from the history, she had symptoms of dyspepsia about 1 year prior to getting pregnant and during the pregnancy she was on antacids.

Sepsis has been described as an etiological factor to acute gastroduodenal ulceration [20,22]. The first patient had features of sepsis which could be a contributory factor. The hematological and biochemical derangements as well as clinical features culminated in a SOFA score of 6 on admission. The association between hypertensive disorders in pregnancy and gastroduodenal perforation is not clear [16]. The first case had pregnancy-induced hypertension and the second case imminent eclampsia.

Symptoms of epigastric pain both present in pre-eclampsia and perforations pose a diagnostic challenge. At the time of diagnosis of imminent eclampsia, the second patient had epigastric pain. Ranganna et al reported a case of duodenal perforation in a nullip with twin gestation with imminent eclampsia in whom the clinical manifestation of the perforation was masked by that of the imminent eclampsia [16]. Perforation leads to chemical peritonitis and this can lead to shock, electrolyte imbalance, sepsis and acute kidney injury all of which may be potentially fatal [13]. The duration from onset of perforation to surgical intervention remains an independent determinant of outcome $[12,23]$. The longer the duration is, the poorer the prognosis with respect to morbidity and mortality [24].

In both cases the time duration of perforations were more than $24 \mathrm{~h}$. The mortality in the first case could be attributed to the prolonged period from perforation to surgical intervention. The patient was likely to have perforated more than 3 days prior to surgical intervention. The complaint of abdominal pain was on postoperative day 5 at the referral site. She had acute kidney injury and sepsis prior to surgical intervention which was instituted on postoperative day 8 after the cesarean section.

In the second case the perforation may have occurred after the exploratory laparotomy for the intraabdominal abscess. There is also the possibility that the perforation occurred earlier prior to the diagnosis of the intraabdominal abscess and laparotomy; however the limitation of inadequate exposure to visualize the epigastric structures may have led to missing the perforation.

The Boey's score in recent times have been used as a predictor of mortality and postoperative complications [7]. The score consisting of prolonged perforation, shock on presentation and confounding medical illness. Two of these were present in the patients; that is compensated shock and the prolonged perforation.

The site of the perforation also has a bearing on outcome. Gastric ulcers in general have been shown to carry an increased mortality risk [3, 4]. However, in this reports both sites of perforation ended up with mortality. The complications of chemical peritonitis from the perforation including shock, electrolyte imbalance, sepsis and acute kidney injury were all evident in both cases. These were significant contributors to the morbidity and mortality [24]. Mortality rates in recent studies of perforation from peptic ulcer disease in the sub re- 
gion range between $8 \%$ and $16.4 \%[12,24]$. The postoperative complications seen in both cases are similar to that described in other case reports and series which are contributors to morbidity and mortality.

\section{Conclusions}

Gastroduodenal perforation carries an increased risk of mortality and this is further compounded by prolonged period between perforation and surgical intervention. It may be necessary to reevaluate the use of NSAIDS in patients undergoing cesarean section that have dyspepsia during pregnancy as well as upper gastrointestinal disorders in hypertensive disorders of pregnancy to prevent their untoward effects. Large case series are required for this evaluation.

\section{Acknowledgments}

The authors would like to acknowledge the workers of the various directories at the Komfo Anokye Teaching Hospital.

\section{Financial Disclosure}

No funding was obtained for this study.

\section{Conflict of Interest}

The authors declare they have no conflict of interest.

\section{Informed Consent}

Informed consent obtained.

\section{Author Contributions}

RAW, CT and JY were involved in data curation, formal analysis, methodology, software, supervision, validation, writing of original draft, writing of review and editing; SA, MOA and EAA were involved in conceptualization, data curation, formal analysis, supervision, writing of original draft, writing of review and editing; KA, SAA and EA contributed in conceptualization, data curation, formal analysis, software, supervision, validation, writing of original draft, writing of review and editing; SV, PAP, DA and DAY were involved in conceptualization, data curation, investigation, writing of original draft, writing of review and editing.

\section{References}

1. Malfertheiner P, Chan FK, McColl KE. Peptic ulcer disease. Lancet. 2009;374(9699):1449-1461.
2. Sung JJ, Kuipers EJ, El-Serag HB. Systematic review: the global incidence and prevalence of peptic ulcer disease. Aliment Pharmacol Ther. 2009;29(9):938-946.

3. Chalya PL, Mabula JB, Koy M, McHembe MD, Jaka HM, Kabangila R, Chandika AB, et al. Clinical profile and outcome of surgical treatment of perforated peptic ulcers in Northwestern Tanzania: A tertiary hospital experience. World J Emerg Surg. 2011;6:31.

4. Svanes C. Trends in perforated peptic ulcer: incidence, etiology, treatment, and prognosis. World J Surg. 2000;24(3):277-283.

5. Rajesh V, Chandra SS, Smile SR. Risk factors predicting operative mortality in perforated peptic ulcer disease. Trop Gastroenterol. 2003;24(3):148-150.

6. Hermansson M, Stael von Holstein C, Zilling T. Surgical approach and prognostic factors after peptic ulcer perforation. Eur J Surg. 1999;165(6):566-572.

7. Boey J, Choi SK, Poon A, Alagaratnam TT. Risk stratification in perforated duodenal ulcers. A prospective validation of predictive factors. Ann Surg. 1987;205(1):2226.

8. Mabogunje OA, Lawrie JH. Surgical management of duodenal ulcer in Zaria, Nigeria. J R Coll Surg Edinb. 1985;30(5):283-287.

9. Otu AA. Prospective evaluation of simple suture in the surgical treatment of perforated peptic ulcer. West Afr J Med. 1990;9(4):295-298.

10. Khan SH, Haq MIU. Perforated peptic ulcer. Aziz SAJTPMJ. 2011;18(01):124-127.

11. Levison ME, Bush LM. Peritonitis and intraperitoneal abscesses. In: Mandell, Douglas, and Bennett's principles and practice of infectious diseases. edn.: Elsevier; 2015: p. 935-959. e934.

12. Dakubo JC, Naaeder SB, Clegg-Lamptey JN. Gastroduodenal peptic ulcer perforation. East Afr Med J. 2009;86(3):100-109.

13. Steenvoorde P, Moues CM, Viersma JH. Gastric perforation due to the ingestion of a hollow toothpick: report of a case. Surg Today. 2002;32(8):731-733.

14. Fletcher MS. Gastric perforation secondary to metastatic carcinoma of the lung: a case report. Cancer. 1980;46(8):1879-1882.

15. Nakao A, Isozaki H, Iwagaki H, Kanagawa T, Takakura N, Tanaka N. Gastric perforation caused by a bulimic attack in an anorexia nervosa patient: report of a case. Surg Today. 2000;30(5):435-437.

16. Ranganna H, Nalini K. Duodenal perforation in a lady with twin gestation and severe preeclampsia. Biliangadi HNJJoCR. 2013;3(2):349-352.

17. Horwich M. Perforated duodenal ulcer during pregnancy. Br Med J. 1958;2(5089):145.

18. Barnes LW. Serum histaminase during pregnancy. Obstet Gynecol. 1957;9(6):730-732.

19. Townsend CM, Beauchamp RD, Evers BM, Mattox KL. Sabiston textbook of surgery: the biological basis of modern surgical practice: Elsevier Health Sciences; 2016.

20. Badoe E, Archampong E, Jaja M. Principles and practice of surgery, including pathology in the tropics: University of Ghana Medical School; 1994. 
21. Laine L: The gastrointestinal effects of nonselective NSAIDs and COX-2-selective inhibitors. In: Seminars in arthritis and rheumatism: 2002: Elsevier; 2002: p. 25-32.

22. Fogelman MJ, Garvey JM. Acute gastroduodenal ulceration incident to surgery and disease. Analysis and review of eighty-eight cases. Am J Surg. 1966;112(5):651-656.

23. Subedi SK, Afaq A, Adhikary S, Niraula SR, Agrawal
CS. Factors influencing mortality in perforated duodenal ulcer following emergency surgical repair. JNMA J Nepal Med Assoc. 2007;46(165):31-35.

24. Nuhu A, Madziga AG, Gali BM. Acute perforated duodenal ulcer in Maiduguri: experience with simple closure and Helicobacter pylori eradication. West Afr J Med. 2009;28(6):384-387. 